\title{
Improving Displacement Number and Overheads of DRFN using Artificial Bee Colony Technique in WSNs
}

\author{
Rajneet Kaur \\ Research Scholar \\ Dept. of computer science \\ DAVIET, Jalandhar
}

\author{
Shaveta Angurala \\ Asst. Professor \\ Dept. of computer science \\ DAVIET, Jalandhar
}

\begin{abstract}
Failure in Wireless Sensor Networks is common due to deployment of sensor nodes in harsh or hostile environment with limited power backup. Node failures could degrade the efficiency of sensor networks. Thus, failure detection and recovery techniques are very crucial for effective performance of nodes in Wireless Sensor Networks. In this paper we presented an improved DRFN technique by improving its failover mechanism. The failure handling scheme will be improved by using Artificial Bee Colony (ABC) based optimization technique. We analyze existing DRFN technique and compare it with the proposed technique on the basis of performance metrics such as occurrence of displacements and displacement overheads. Experimental results show that the proposed failure detection and recovery technique outperform over the available technique. The presented scheme is implemented and analyzed in 2013 version of MATLAB simulation tool.
\end{abstract}

\section{Keywords}

Artificial Bee Colony (ABC) based Optimization, Failure handling, Mobile nodes, Wireless sensor networks (WSN).

\section{INTRODUCTION}

In the last decade, Wireless Sensor Networks (WSNs) have emerged as a promising technology with growing interest in many applications areas such as space exploration, border and coastal security [2], [6],[15]. A wireless sensor network contains small sensor nodes that are placed over a large geographical area in order to monitor various physical phenomena like vibrations, temperature, seismic events, humidity and so on [3].Sensor nodes are small in size, wirelessly communicating, where each node has a power battery, sensing range, communication ability and computation engine [19]. Base station is called sink node that are responsible for data collection from sensor nodes. Internet facility is used by base station for sending data to end users for further processing [13].

Sensor networks provide endless applications, such as monitoring environments, traffic movements and transportation, military observations, medical, and agronomic scientific etc. but at the same time provide forbidding challenges such as scarcity of energy [16]. Due to limited battery lifetime and low energy, sensors are in danger to different failures [18]. Faulty sensor nodes are one of the most important sources of faults [7]. The issue of failures handling in WSNs is very crucial, since nodes are often deployed in a harsh environment for example battlefield surveillance, fire detection, volcano or glacier monitoring and are prone to unexpected changes which may harm the normal operation of the WSNs[5],[8]. In this paper, we focus on improving DRFN technique by improving its failover scheme according to the principles of swarm intelligence by applying Artificial Bee Colony Based Optimization technique.

Swarm intelligence (SI) is a relatively novel field that generally refers to the study of the collective behavior of multi-component systems that coordinate using decentralized controls and self-organization [14].In recent years, a new swarm intelligence-based optimization algorithm, called Artificial Bee Colony ( $\mathrm{ABC}$ ), has greatly attracted the attention of researchers [10]. Swarm intelligence contain a new member known as Artificial Bee Colony (ABC) technique. This technique has been applied to various problems such as in optimization of numerical problems, data clustering, wireless sensor network deployment and routing [12],[17].In this paper Artificial Bee Colony technique is applied to find optimum path between sender \& base station and DRFN technique is applied in case of failure occur in the route obtained by $\mathrm{ABC}$ technique .

The remainder of the paper is organized as follows: Section II presents the related work. Section III presents the proposed algorithm and section IV describes the experimental results. Finally, section V concludes the paper.

\section{RELATED WORK}

In [21] this paper proposed a fault tolerant routing which involves fault recovery process with fault detection scheme, referred to as energy efficient fault tolerant multipath routing scheme for wireless sensor network (FTMRS). In FTMRS technique every sensor node transmits its data to a base station through shortest path. The performance analysis of FTMRS shows better results compared to other popular fault tolerant techniques in wireless sensor networks. In [23] this paper, proposed a novel fault-tolerant distributed multiclass classification fusion approach using error correcting codes (DCFECC) that provides excellent fault-tolerance capability in WSN. This new approach not only provides an improved fault-tolerance capability but also reduces computation time and memory requirements at the fusion center. In [24] this paper, we address the issues of maintaining sensing coverage and connectivity by keeping a minimum number of sensor nodes in the active mode in wireless sensor networks. Based on the optimality conditions, we then devise a decentralized density control algorithm, OGDC (Optimal Geographical Density Control), for density control in large scale sensor networks. [9] proposed a Detection and Replacement of a Failing Node approach for the connectivity maintenance by carrying out a replacement chain according to a distributed algorithm. The main goal of this method is to restore the connectivity of network by using the sensors mobility taking into account the energy constraint. Result shows that the presented method consumes less energy, and improves the percentage of reduction in field coverage as compared to other approaches. [4] proposed a Cluster based Wireless sensor 
network routings using Artificial bee colony algorithm. Result shows that complexity analysis of presented strategy outperforms over direct transmission and LEACH algorithm. [11] presented $\mathrm{ABC}$ with real coded crossover operator applied to minimize the length of the tour and find the optimal path. Hence $\mathrm{ABC}$ algorithm is used to find shortest path in travelling salesman problem. The experimental result shows that proposed algorithm provides better accuracy and efficiency than the Spanning tree covering algorithm .[20] deals with reducing the make span of the job scheduling task to the minimum by employing Artificial Bee Colony algorithm. In contrast to GA (genetic algorithm), ABC algorithm produced better results.[22] proposed an enhanced DRFN technique by enhancing its failover scheme. The failover scheme will be enhanced using Artificial Bee Colony $(\mathrm{ABC})$ based optimization. It will reduce the overall energy consumption further, and also reduce the number of displacements occur in order to replace the failed node.A novel coverage conscious connectivity restoration (C3R) algorithm is presented in [1]. C3R involves one or multiple neighbors of the failed node to recover from the failure. Each neighbor of the failed node temporarily moves in order to substitute later. Each neighbor involved in the procedure of recovery moved from its original position towards the failed node position in order to perform function of failed node. Energy-centric optimized recovery algorithm (ECR) is also introduced for energy efficiency.

\section{PROPOSED ALGORITHM}

This section includes the steps of the proposed technique. It presents the methodology of presented algorithm with its steps. In proposed algorithm, firstly sensor nodes are deployed in a square area randomly in Wireless Sensor Networks. In second step, Artificial Bee Colony technique is applied in order to find best or shortest path between sender node and base station. $\mathrm{ABC}$ algorithm involve various phases: initialization phase, employed bee phase, onlooker bee phase and scout bee phase. After this step, DRFN technique is applied in case of occurrence of failure of sensor node in the path discovered by Artificial Bee Colony technique. In fourth step of proposed algorithm after failure is detected and removed, rerouting technique is applied to obtain alternative route using Artificial Bee Colony technique. In last step, all the required parameters are evaluated which involve: number of displacements and displacement overhead. The main phases of $\mathrm{ABC}$ algorithm are as follows:

i. Initialization: All routes are initialized in the range $\left[l_{j}, u_{j}\right]$ by Eq. (1)

$$
x_{i j}=l_{j}+\operatorname{rand}(0,1)\left(u_{j}-l_{j}\right)
$$

Where $l_{j}$ and $u_{j}$ is lower and upper bound of route $x_{i j}$ and $\operatorname{rand}(0,1)$ is a random number between 0 and 1 .

ii. Employed bees Phase :Each employed bee is associated with the route and determine new path in its neighborhood by Eq. (2)

$$
n_{i j}=x_{i j}+\varphi_{i j}\left(x_{i j}-x_{k j}\right)
$$

Where $\mathrm{j}$ is a randomly chosen parameter and $\mathrm{k}$ is a randomly chosen solution different from $\mathrm{i}$ and $\varphi_{i j}$ is random number within $[-1,1]$.

iii. Onlooker bees phase : onlooker bees are probabilistically moved to the routes based on the following Eq.(3)

$$
\operatorname{prob}_{i}=\frac{\mathrm{fit}_{i}}{\sum_{j=1}^{s n} \text { fit }_{i}}
$$

Where $\operatorname{prob}_{i}$ refers to probability of the $x_{i}$ route to be selected, $s n$ is the number of routes and fitness $s_{i}$ is the fitness value of the route $x_{i}$.

iv. Scout bee phase: Randomly initialize path by a scout bee using Eq. (1) 


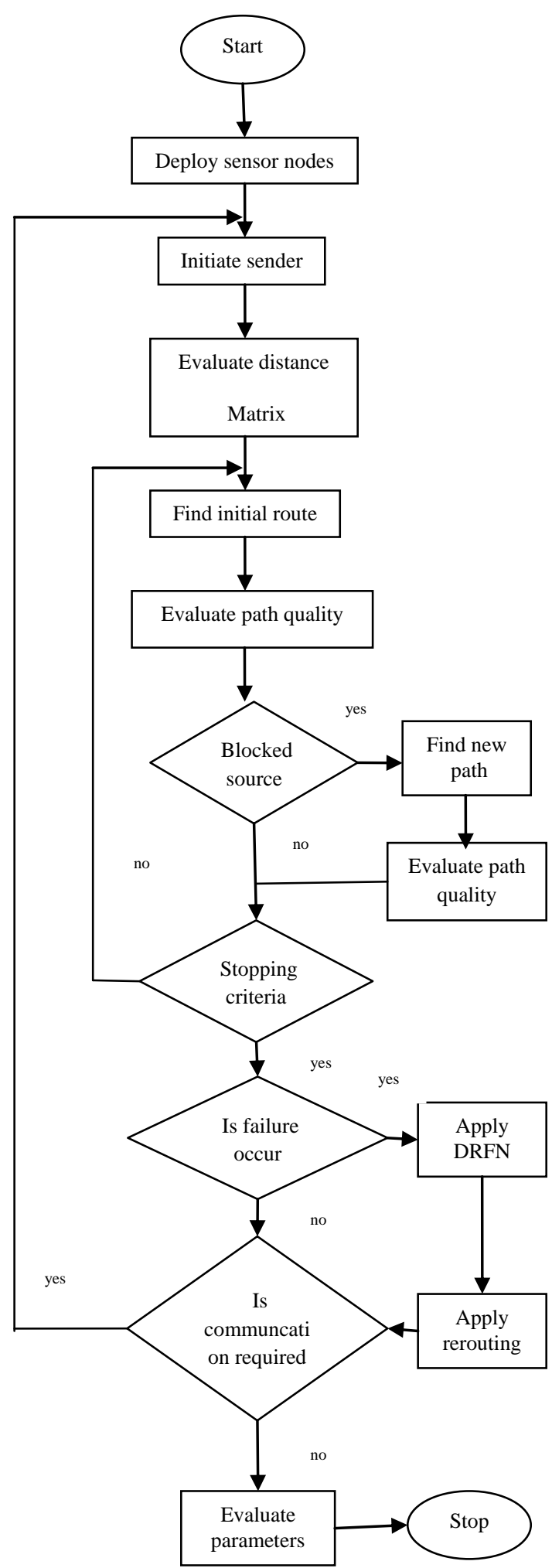

Fig.1 Flowchart of proposed algorithm

\section{EXPERIMENTAL RESULTS}

Simulation in MATLAB is done to determine the performance of ABC-DRFN algorithm. In this simulation, a set of mobile sensor nodes are placed randomly in a $100 \times 100 \mathrm{~m}^{2}$ area along $\mathrm{x}$-axis and $\mathrm{y}$-axis to form Wireless Sensor Network.

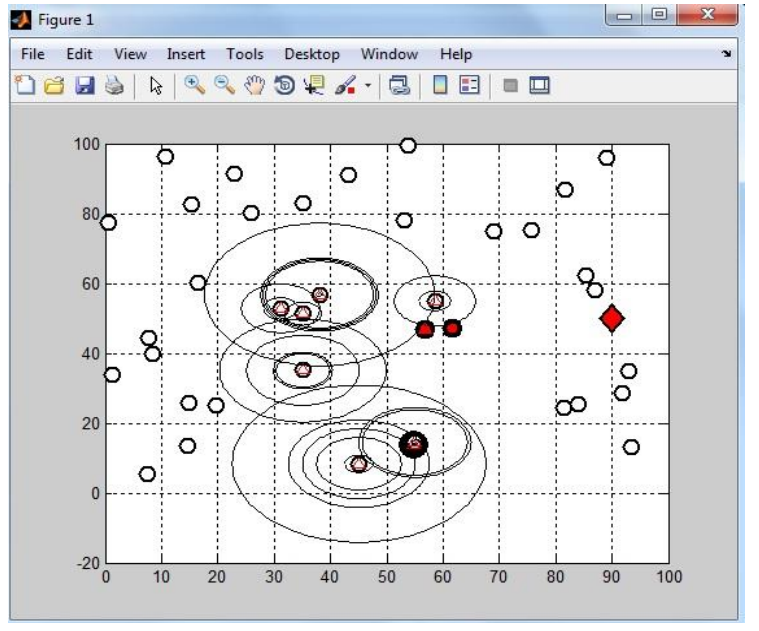

Fig.2 Self-Organized Data Collecting and Transmitting Phase of DRFN

In this, proposed algorithm performance is compared with existing DRFN algorithm after evaluation of parameters that includes: number of displacements and displacement overheads.

i. Number of displacements: Comparison of displacement number between ABC-DRFN and DRFN is shown in Table 1.From graph shown in Figure 3, it is clear that number of displacements in ABC-DRFN is less than number of displacements in DRFN where $\mathrm{x}$-axis represents number of rounds and y-axis represents number of displacements.

Table.1 Comparision of displacements number in DRFN and ABC-DRFN.

\begin{tabular}{|c|c|c|}
\hline Rounds & DRFN & ABC-DRFN \\
\hline 15 & 3.0000 & 2.4000 \\
\hline 25 & 4.6800 & 3.6400 \\
\hline 35 & 6.3429 & 4.8857 \\
\hline 45 & 8.0000 & 6.1333 \\
\hline 55 & 9.6727 & 7.3818 \\
\hline 65 & 11.3385 & 8.6308 \\
\hline 75 & 13.0000 & 9.8800 \\
\hline 85 & 14.6766 & 11.1294 \\
\hline 95 & 16.3368 & 12.3789 \\
\hline
\end{tabular}

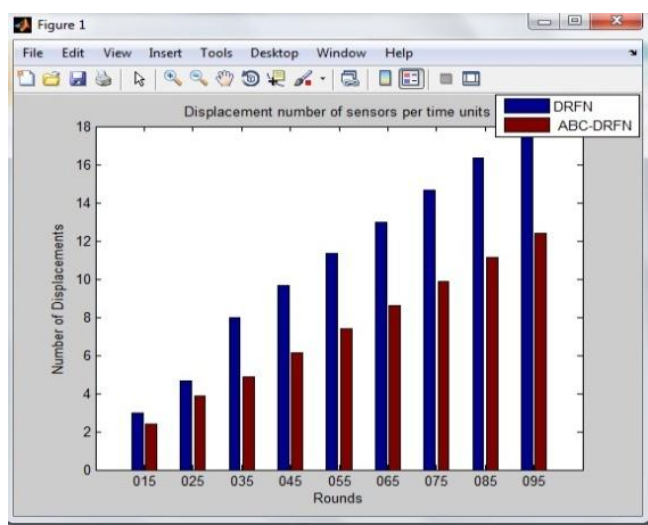

Fig.3 Number of displacements in DRFN and ABC-DRFN. 
ii. Displacement overheads: Comparison of displacement overhead between ABC-DRFN and DRFN is shown in Table 2.From graph shown in Figure 4, it is clear that displacement overhead occur in ABC-DRFN is less than overheads occur in DRFN where $\mathrm{x}$-axis represents number of rounds and $y$-axis represents displacements overheads.

Table.2 Comparision of displacement overheads in DRFN and $A B C$-DRFN.

\begin{tabular}{|c|c|c|}
\hline Rounds & DRFN & ABC-DRFN \\
\hline 15 & 0.0632 & 0.0546 \\
\hline 25 & 0.1405 & 0.0714 \\
\hline 35 & 0.1105 & 0.0858 \\
\hline 45 & 0.1879 & 0.1296 \\
\hline 55 & 0.3320 & 0.2466 \\
\hline 65 & 0.1950 & 0.1625 \\
\hline 75 & 0.1972 & 0.1612 \\
\hline 85 & 0.2625 & 0.1915 \\
\hline 95 & 0.2895 & 0.2396 \\
\hline
\end{tabular}

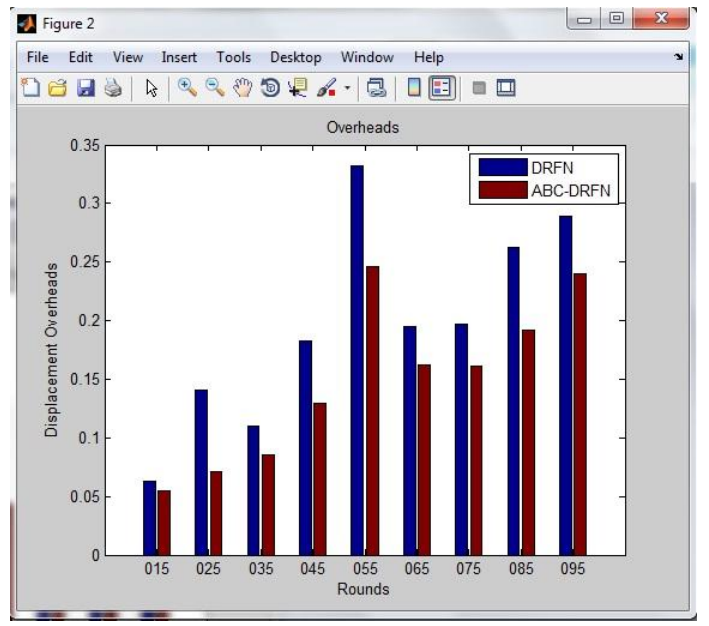

Fig.4 Displacement overheads in DRFN and ABC-DRFN.

\section{CONCLUSION}

Sensor nodes in Wireless Sensor Networks may fail due to limitation of energy or less power supply and harsh environment in which it is deployed. In this paper we proposed an improved DRFN technique by improving its failover mechanism. The failure handling mechanism would be improved by using Artificial Bee Colony (ABC) based optimization technique. The presented technique has decreased the occurrence of displacements and also reduces displacement overheads. Result of simulation shows that the proposed technique performs better than existing technique.

\section{REFERENCES}

[1] N.Tamboli and M. Younis, "Coverage-aware connectivity restoration in mobile sensor networks", Journal of Network and Computer Applications, Vol.33,Issue 4,pp.363-374, 2010.

[2] Sayaka Kamai, Takashi Nagai and Satoshi Fujita,"Fast and reliable maintenance protocols for WSN with crash and intermittent failures", In IEEE 2nd International Conference on Networking and Computing, Osaka, pp. $40-49,2011$.
[3] G. Anastasi, M. Conti, M. D. Francesco and A. Passarella," Energy conservation in wireless sensor networks: A survey", In journal of Ad Hoc Networks , pp.537-568, 2009.

[4] S. Okdem, D.Karaboga and C. Ozturk,“ An Application of Wireless Sensor Network Routing based on Artificial Bee Colony Algorithm", In IEEE Congress on Evolutionary Computation, New Orleans, LA, pp.326330,2011

[5] M.Aliouat, Z.Aliouat and M.Naidja,"Adaptative Nodes Diagnosis and Recovery for wireless sensor networks", In IEEE International Sympsium on computer applications and Industrial Electronics, Kota Kinabalu Malaysia, pp.256 - 261, 2012.

[6] M.Bellalouna and A.Ghabri,“A Priori methods for Fault Tolerance in wireless Sensor Networks", In IEEE World Congress on Computer and Information Technology, Sousse, pp.1-6, 2013.

[7] S. Jia, W. Bailing and P. Xiyuan,“ An Efficient Recovery Algorithm for Coverage Hole in WSNs", American Society of Transportation \& Logistics, Vol. 18, pp. 5-9, 2013.

[8] H.Redwan,G.Akele and Ki-Hyung Kim, "Cluster-Based Failure Detection and Recovery Scheme in Wireless Sensor Network", In IEEE Sixth International Conference on Ubiquitous and Future Networks, Shanghai, pp.407-412, 2014.

[9] A.Boundries ,M.Aliouat and P.Siarry, "Detection and Replacement of a failing node in the wireless sensor networks ", International journal of Computers \& Electrical Engineering, Vol. 40, Issue 2, pp.421-432, 2014.

[10] D.Jia, X.Duan and M.K.Khan, "Binary Artificial Bee Colony optimization using bitwise operation", International journal of Computers \& Industrial Engineering, Vol.76, Issue 2, pp.360-365, 2014.

[11] R.Selva Bharathil ,R.Priyadharshni and S.Ramesh,“ Artificial Bee Colony Algorithm to Find Optimum Path for Mobile Agents in Wireless Sensor Networks", In IEEE International Conference on Advanced Communication Control and Computing Technologies, Ramanathapuram, pp.842-846, 2014.

[12] . Ozturk, E. Hancer, D. Karaboga, "Dynamic Clustering with Improved Binary Artificial Bee Colony Algorithm", Applied Soft Computing Journal, Vol.28, pp.6980,2015 .

[13] R. Kaur and S. Angurala, "Improved Rendezvous Nodes Based LEACH Using Hybrid PSO/GA", In International Journal of Engineering and Innovative Technology (IJEIT), Vol. 4, Issue 10, pp.85-89, April 2015.

[14] M. Saleem,Gianni A.Di Caro and M.Farooq, "Swarm intelligence based routing protocol for wireless sensor networks: Survey and future directions", Journal of Information Sciences, Vol. 181,Issue 20,pp.4597-4624, 2010.

[15] M. Younis, I. F. Senturk, K. Akkaya, S.Lee and F. Senel, "Topology management techniques for tolerating node failures in wireless sensor networks: A survey", In journal of Computer Networks, pp.254-283, 2014. 
[16] D. Puccinelli and M.Haenggi, "Wireless Sensor Networks: Applications and Challenges of Ubiquitous Sensing”, IEEE Circuits and Systems Magazine, 2005.

[17] A. Baykaso, L. Özbakır and Pınar Tapkan, "Artificial Bee Colony Algorithm and Its Application to Generalized Assignment Problem", Open Access Database www.i-techonline.com.

[18] I.F. Senturk, K. Akkaya and S.Yilmaz," Relay placement for restoring connectivity in partitioned wireless sensor networks under limited information", In journal of Ad Hoc Networks, pp.487-503, 2014.

[19] F. Koushanfar, M. Potkonjak and A. S. Vincentelli, “ Fault Tolerance in Wireless Sensor Networks", wwwece.rice.edu/ fk1/papers/chapter-FT_04.pdf

[20] A.Muthiah and R.Rajkumar, "A Comparison of Artificial Bee Colony algorithm and Genetic Algorithm to minimize the makespan for Job Shop Scheduling",12th
Global Congress on Manufacturing and Management, Vol .97, pp.1745-1754, 2014

[21] P.Chanak, T.Samanta and I.Banerjee, "Fault-Tolerant Multipath Routing Scheme for energy efficient Wireless Sensor Networks", International Journal of Wireless \& Mobile Networks (IJWMN), Vol. 5, No. 2, April 2013.

[22] Rajneet Kaur and Shaveta Angurala,“ Enhanced DRFN Failover Scheme Using Artificial Bee Colony Based Optimization in Wireless Sensor Networks", International Journal of Engineering and Innovative Technology (IJEIT), Vol 5, Issue 1, pp.59-63, 2015

[23] Tsang-Yi Wang, Yunghsiang S. Han, Pramod K. Varshney and Po-Ning Chen, "Distributed Fault-Tolerant Classification in Wireless Sensor Networks", IEEE Journal on selected areas in communications, Vol. 23, No. 4, April 2005.

[24] H. Zhang and J. C. Hou, "Maintaining Sensing Coverage and Connectivity in Large Sensor Networks", Ad Hoc \& Sensor Wireless Networks , pp. 89-124, 2005. 